\title{
Análisis de la percepción de seguridad en puentes peatonales: una aproximación mediante modelación híbrida*
}

Luis Márquez ${ }^{* *}$

Recibido: 10/10/2014 • Aceptado: 11/05/2015

\begin{abstract}
Resumen
Este artículo de investigación científica y tecnológica estudia la percepción de seguridad en el uso de puentes peatonales, empleando un enfoque sustentado en dos campos principales: el microeconómico y el psicológico. El trabajo hace la estimación simultánea de un modelo híbrido de elección y variables latentes con datos de una encuesta de preferencias declaradas, encontrando mejor ajuste que un modelo mixto de referencia, lo que indica que la percepción de seguridad determina el comportamiento de los peatones cuando se enfrentan a la decisión de usar o no un puente peatonal. Se encontró que el sexo, la edad y el nivel de estudios son atributos que inciden en la percepción de seguridad. El modelo calibrado sugiere varias estrategias para aumentar el uso de puentes peatonales que son discutidas, encontrando que el uso de barreras ocasiona una pérdida de utilidad, en los peatones, que debería ser estudiada como extensión del presente trabajo.

Palabras clave: modelos de elección discreta, variables latentes, modelación híbrida, percepciones de seguridad.
\end{abstract}

El presente trabajo de investigación se deriva de la comisión remunerada para adelantar estudios de Doctorado en Ingeniería Civil en la Universidad del Norte de Barranquilla, según contrato 0344-2013.

** Profesor asociado de la Universidad Pedagógica y Tecnológica de Colombia, magíster en Ingeniería con énfasis en Transporte, Especialista en Transporte Urbano, Especialista en Gobierno Municipal. Avenida Central del Norte 39-115, Tunja, Colombia. (57) 8 7405626. luis.marquez@uptc.edu.co 


\title{
Analysis of safety perception on pedestrian bridges: a hybrid modelling approach
}

\begin{abstract}
This article of scientific and technological research studies safety perception on pedestrian bridges, through an approach based on two main areas: the microeconomic and psychological. This paper estimates simultaneously a hybrid model of choice and latent variables, with data from a stated preference survey, finding that this model fits better than a mixed logit reference model, which indicates that safety perception determines the behaviour of pedestrians when faced with the decision to use or not a pedestrian bridge. It was found that gender, age and educational level are attributes that affect safety perception. The calibrated model suggests several strategies to increase the use of pedestrian bridges, which are discussed, finding that the use of pedestrian barriers causes a loss of utility that should be studied as an extension of this work.
\end{abstract}

Key words: Discrete choice models, latent variables, hybrid modelling, safety perceptions. 


\section{INTRODUCCIÓN}

El comportamiento de los peatones puede entenderse como la manifestación de un proceso complejo, condicionado por factores individuales, atributos del viaje y características del entorno. Experiencias previas, presencia de otros peatones e información del riesgo determinan la conducta de los peatones, que puede cambiar en función del propósito, el horario, el origen, el destino o la longitud de la caminata, entre otros atributos del viaje. También hay una influencia importante del patrón de flujos vehiculares y de la existencia y diseño de las facilidades peatonales. Desde esta perspectiva, el comportamiento de los peatones es un proceso difícil de modelar.

A pesar de la dificultad inherente, la modelación de los desplazamientos peatonales en el entorno urbano de las ciudades ha sido de gran interés para la comunidad científica mundial [1]; sin embargo, en el contexto de los estudios de movilidad urbana realizados en Colombia, basados normalmente en encuestas domiciliarias, el análisis de los desplazamientos peatonales no se aborda con el mismo rigor aplicado en el estudio de los viajes motorizados. Por tal razón, modelar las variaciones de actitud de los individuos [2-3], incorporando, además, atributos del entorno del cual el individuo es parte [4] es un reto de gran importancia que vale la pena asumir.

En las últimas décadas se han desarrollado diferentes modelos y teorías para tratar de explicar las elecciones que realiza cada miembro de la sociedad cuando interviene en el sistema de transporte [5]. Dichas teorías se ubican en dos campos principales: el microeconómico y el psicológico. En el campo microeconómico se han aplicado los modelos de elección discreta que se basan en la maximización de la utilidad aleatoria, siendo los modelos mixtos los de mayor flexibilidad. Los psicólogos, en cambio, han elaborado teorías del comportamiento más ajustadas a las decisiones reales pero de menor aplicación práctica. Los esfuerzos realizados por aproximar estos dos campos han dado como resultado la modelación híbrida.

Se considera que los modelos híbridos son superiores incluso a los modelos tradicionales de gran flexibilidad, como es el caso de los modelos mixtos, ya que estos últimos ignoran el efecto de las actitudes y percepciones subjetivas [6]. La modelación híbrida ha sido utilizada con buenos resultados en distintos contextos de elección que han incorporado el efecto de variables latentes como la percepción de seguridad, siendo posible destacar, entre otros, el análisis de la aversión al riesgo en el comportamiento de viajeros [7], el efecto de la seguridad en la compra de vehículos [8], las preocupaciones de los usuarios de tren [9], el análisis de variables latentes en la elección de ruta [10] y el estudio de percepciones de comodidad y seguridad en el transporte fluvial [11].

Es importante resaltar cómo algunos estudios que han investigado las políticas de transporte sin tomar en cuenta las percepciones de los usuarios han llegado a la 
conclusión de que se debe analizar el comportamiento de los individuos para mejorar la gestión de dichas políticas [12]. En esta línea, han sido analizadas las percepciones de distintas clases de individuos con respecto a las políticas tomadas en materia de infraestructura [13] y en el uso de servicios de transporte público, y se ha encontrado que las percepciones de seguridad tienen un significativo efecto sobre el uso del servicio [14].

En el marco de los modelos híbridos como herramienta de investigación y análisis de políticas de transporte, el presente trabajo estudia la percepción de seguridad en el uso de puentes peatonales. El enfoque metodológico involucró diseñar y aplicar encuestas para la estimación de modelos de elección incluyendo variables latentes, a fin de estudiar el efecto de la percepción de seguridad sobre el comportamiento de los peatones al momento de cruzar una vía dotada con infraestructura peatonal. Específicamente se usaron datos provenientes de una encuesta de preferencias declaradas, aplicada a peatones, usuarios y no usuarios de puentes peatonales en la ciudad de Tunja [15].

\section{CONTEXTO}

Tunja, capital del departamento de Boyacá, localizada $131 \mathrm{~km}$ al norte de Bogotá, tiene actualmente 173,216 habitantes. La ciudad ha tenido un importante desarrollo urbano en los últimos años y se caracteriza por ser una urbe de servicios, con una fuerte vocación educativa, tanto así que comúnmente se le denomina "ciudad universitaria". Tunja ha crecido longitudinalmente a lado y lado de los ejes de las avenidas Norte y Oriental, que además del tráfico urbano reciben buena parte del tráfico regional, especialmente buses intermunicipales que deben transitar por estas vías para acceder a la terminal de transporte. Esta dinámica de crecimiento ha determinado la localización de puentes peatonales en la ciudad, prácticamente todos ubicados sobre estas dos importantes avenidas.

La ciudad cuenta con 8 puentes peatonales que se encuentran localizados sobre tres vías principales: avenida Norte, avenida Maldonado y avenida Oriental, como se puede ver en la tabla 1. En general los puentes peatonales de la ciudad son relativamente cortos y todos ellos están ubicados sobre vías de doble calzada con separador, con 2 carriles de circulación por sentido. Los mayores volúmenes horarios se presentan en la avenida Norte y en la avenida Maldonado. El uso de los puentes peatonales de la ciudad es de aproximadamente $60 \%$, lo que indica que por cada 10 personas que cruzan estas avenidas, en los sitios donde están ubicados los puentes peatonales, únicamente 6 hacen uso de ellos. Los puentes más utilizados son el de la Universidad Pedagógica y Tecnológica de Colombia-UPTC-, que tiene una malla divisoria en el separador, y el de Los Lanceros, que tiene acceso a nivel por estar localizado justo sobre el talud de la vía, lo que evita el uso de las escaleras en uno de sus extremos. 
Tabla 1. Ubicación de puentes y porcentaje de uso

\begin{tabular}{|c|c|c|c|c|c|c|c|}
\hline$N .^{\circ}$ & Puente & $\begin{array}{c}\text { Longitud } \\
\text { (m) }\end{array}$ & Ubicación & $\begin{array}{l}\text { Volumen } \\
\text { Horario } \\
\text { máximo }\end{array}$ & $\begin{array}{c}\text { Velocidad } \\
\text { vehicular } \\
(\mathrm{km} / \mathrm{h})\end{array}$ & $\begin{array}{l}\% \text { uso } \\
\text { puente }\end{array}$ & $\begin{array}{l}\% \text { no uso } \\
\text { Puente }\end{array}$ \\
\hline 1 & Santa Rita & 26.6 & \multirow{3}{*}{ Avenida Norte } & 920 & 46.9 & 27 & 73 \\
\hline 2 & Santa Inés & 32.4 & & 1,788 & 58.0 & 50 & 50 \\
\hline 3 & UPTC & 24.4 & & 1,788 & 52.5 & 95 & 5 \\
\hline 4 & Maldonado & 21.9 & Avenida Maldonado & 1,360 & 32.0 & 51 & 49 \\
\hline 5 & Colboy & 22.5 & \multirow{4}{*}{ Avenida Oriental } & 433 & 44.2 & 43 & 57 \\
\hline 6 & Los Lanceros & 36.6 & & 521 & 56.4 & 100 & 0 \\
\hline 7 & Jordán Hunza & 25.0 & & 668 & 42.3 & 17 & 83 \\
\hline 8 & Santiago de Tunja & 25.8 & & 719 & 40.5 & 85 & 15 \\
\hline
\end{tabular}

Fuente: elaboración propia

En Tunja la tasa de muertes en accidentes de tránsito es de 16.86 por cada 100,000 habitantes, es decir 30 muertes al año: 22 hombres y 8 mujeres. En cuanto a los lesionados, la tasa es de 152.27, que corresponde a 271 heridos al año: 170 hombres y 101 mujeres [16], siendo en general los peatones, pasajeros y motociclistas las víctimas más frecuentes. Las vías de mayor ocurrencia de víctimas fatales son las avenidas Oriental y Norte. Sobre la avenida Oriental se destacan lugares específicos como Los Hongos y las inmediaciones de la terminal de transporte. En el caso de la avenida Norte, los sitios críticos se localizan en los sectores de La Glorieta, Olímpica y el barrio Los Muiscas [17].

\section{METODOLOGÍA}

El trabajo de investigación se sustentó en la estimación simultánea de un modelo híbrido de elección y variables latentes, con base en una encuesta de preferencias declaradas, aplicada a peatones, usuarios y no usuarios de puentes peatonales en la ciudad de Tunja.

Aunque se consideró inicialmente el uso de encuestas de preferencias reveladas, se decidió emplear encuestas de preferencias declaradas, ya que en reuniones focales, previas al diseño de los instrumentos de medición, fueron identificadas dos variables relevantes en el proceso de elección que no eran fáciles de tratar con preferencias reveladas: la accidentalidad y el flujo vehicular.

Básicamente se intentó aprovechar las ventajas inherentes a la experimentación con preferencias declaradas [19], en el sentido de ampliar el rango de variación y construir escenarios que evitaran la existencia de correlación entre estas dos variables. Además, fue posible aislar el efecto de ciertos atributos y considerar variables latentes. 


\subsection{Encuesta}

Para evaluar la percepción de seguridad en el uso de puentes peatonales se diseñó y aplicó una encuesta dirigida a peatones de la ciudad de Tunja, usuarios y no usuarios de los puentes peatonales. El instrumento de toma de información se dividió en tres partes principales: primero se identificó a cada uno de los individuos encuestados, luego se formularon unas preguntas a manera de indicadores de la percepción de seguridad y finalmente se presentó el juego del experimento de elección con base en técnicas de preferencias declaradas.

\section{Descripción de la muestra}

Se aplicó un total de 200 encuestas, repartidas en forma equitativa en los 8 puentes de la ciudad, a razón de 25 encuestas por puente. Se encontró un total de 47 individuos cautivos o lexicográficos, cuyas observaciones no hicieron parte de los datos con los que fueron calibrados los modelos, quedando entonces 153 encuestas útiles, para una muestra total conformada por 612 pseudo-individuos, ya que cada entrevistado se enfrentó a 4 situaciones de elección diferentes.

El $71 \%$ de población encuestada fue menor a 40 años, lo que se considera consistente con la realidad de la ciudad de Tunja, pues buena parte de su población actual es estudiantil. Además, la mitad de los puentes peatonales están localizados en zonas estudiantiles, como es el caso de los puentes Santa Rita, UPTC, Maldonado y Colboy. La distribución por género de la muestra fue $53 \%$ mujeres y $47 \%$ hombres. Se determinó, además, que el $74 \%$ de la muestra cuenta con un nivel de estudios entre bachillerato y universitario, siendo predominante el universitario. El $42 \%$ de los encuestados trabaja, ya sea como independiente o empleado, el $48 \%$ estudia y el $10 \%$ manifestó no estar realizando ningún tipo de actividad.

Indicadores de seguridad

Las variables latentes representan características de los individuos [20, 21] y son incorporadas en un modelo de medición que relaciona las variables latentes con los indicadores observados en un sistema de ecuaciones estructurales. Estadísticamente los indicadores tienen la particularidad de ser endógenos al proceso de elección y, por lo tanto, no son utilizados por el modelo en modo predictivo [22]. Los indicadores son introducidos para caracterizar las variables latentes no observadas y, econométricamente, permitir su identificación, proporcionando, además, mayor eficiencia en la estimación del modelo completo [23]. Así, los indicadores de variables latentes son propuestos en las encuestas como preguntas o afirmaciones con respecto a actitudes, percepciones y memoria. 
Por tal razón la encuesta incluyó 4 preguntas a manera de indicadores con la idea de estudiar la percepción de seguridad, además de los atributos individuales, tal como se ve en la figura 1. La manera como se codificaron los indicadores busca que se dirija hacia la derecha una mayor percepción de seguridad, lo que es importante en términos de la interpretación que se pueda dar a los resultados de la calibración del modelo.

\begin{tabular}{|l|c|c|c|}
\hline \multirow{2}{*}{ Percepción de seguridad } & \multicolumn{3}{|c|}{ Mayor $\gg$ Menor } \\
\cline { 2 - 4 } $\begin{array}{l}\text { Frecuencia con la que camina por el } \\
\text { área de influencia del puente }\end{array}$ & $\begin{array}{c}1 . \\
\text { Casi nunca }\end{array}$ & $\begin{array}{c}2 . \\
\text { Ocasionalmente }\end{array}$ & $\begin{array}{c}3 . \\
\text { Siempre }\end{array}$ \\
\hline $\begin{array}{l}\text { Frecuencia con la que usa el puente } \\
\text { peatonal }\end{array}$ & $\begin{array}{c}1 . \\
\text { Casi nunca }\end{array}$ & $\begin{array}{c}2 . \\
\text { Ocasionalmente }\end{array}$ & $\begin{array}{c}3 . \\
\text { Siempre }\end{array}$ \\
\hline $\begin{array}{l}\text { Motivo por el que usa el puente } \\
\text { peatonal }\end{array}$ & \multicolumn{2}{|c|}{$\begin{array}{c}2 . \\
\text { Otro }\end{array}$} & $\begin{array}{c}2 . \\
\text { Seguridad }\end{array}$ \\
\hline $\begin{array}{l}\text { Sensación de seguridad que genera el } \\
\text { puente peatonal }\end{array}$ & \multicolumn{2}{|c|}{$\begin{array}{c}2 . \\
\text { Baja }\end{array}$} & $\begin{array}{c}3 . \\
\text { Aceptable }\end{array}$ \\
\hline
\end{tabular}

Figura 1. Indicadores de seguridad de la encuesta.

Fuente: elaboración propia

Con respecto a la sensación de seguridad generada por el puente peatonal se encontró que el $60 \%$ de la muestra encuestada tiene una sensación de seguridad aceptable, el $24 \%$ baja, mientras que solo el $16 \%$ de la muestra manifestó tener una sensación de seguridad alta. Aunque no se hicieron indagaciones adicionales al respecto, es posible que la baja sensación de seguridad tenga su origen en problemas de seguridad social, entre los que se pueden citar los robos que eventualmente se presentan en los puentes peatonales.

\section{Diseño experimental}

Se empleó un diseño experimental de preferencias declaradas etiquetadas para dos alternativas: usar el puente peatonal y no usarlo. Cada alternativa se caracterizó con los atributos tiempo y accidentalidad, haciendo variar el tráfico de manera genérica para dar contexto a la elección, tal como se ve en la tabla 2. La idea de haber asociado la accidentalidad en forma específica a cada alternativa fue dar a entender a los entrevistados los distintos niveles de riesgo implícitos en la decisión de usar el puente o cruzar la calle. 
Tabla 2. Alternativas, atributos y niveles del diseño experimental

\begin{tabular}{|l|c|c|}
\hline \multirow{2}{*}{ Atributos } & Usando el puente peatonal & Cruzando por la calle \\
\hline \multirow{2}{*}{ Tiempo (s) } & 30 & 20 \\
\cline { 2 - 3 } & 60 & 40 \\
\hline \multirow{3}{*}{ Accidentes por año } & 5 & 8 \\
\hline \multirow{3}{*}{ Tráfico (v/h) } & 3 & 10 \\
\cline { 2 - 3 } & \multicolumn{2}{|c|}{600} \\
\cline { 2 - 3 } & \multicolumn{2}{|c|}{1,000} \\
\cline { 2 - 3 } & \multicolumn{2}{|c|}{} \\
\cline { 2 - 3 } & \multicolumn{2}{|c|}{1,000} \\
\hline
\end{tabular}

Fuente: elaboración propia

Posteriormente se hizo el diseño experimental, partiendo de un diseño ortogonal, a partir del cual se realizó la configuración de tratamientos a presentar a los individuos encuestados, tal como se muestra en la tabla 3. El diseño experimental definitivo fue el producto de los ajustes realizados con base en la aplicación de dos pruebas piloto, una en octubre del 2012 y otra en marzo de 2013.

Tabla 3. Diseño de la encuesta de preferencias declaradas

\begin{tabular}{|c|c|c|c|c|}
\hline \multicolumn{2}{|c|}{ Usa el puente peatonal } & \multicolumn{2}{c|}{ Cruza por la calle } & \multirow{2}{*}{ Tráfico } \\
\hline Tiempo & Accidentalidad & Tiempo & Accidentalidad & 600 \\
\hline 30 & 5 & 20 & 8 & 800 \\
\hline 30 & 5 & 20 & 8 & 1,000 \\
\hline 30 & 5 & 20 & 8 & 600 \\
\hline 30 & 3 & 20 & 10 & 800 \\
\hline 30 & 3 & 20 & 10 & 1,000 \\
\hline 30 & 3 & 20 & 10 & 600 \\
\hline 60 & 5 & 40 & 10 & 800 \\
\hline 60 & 5 & 40 & 10 & 1,000 \\
\hline 60 & 5 & 40 & 10 & 600 \\
\hline 60 & 3 & 40 & 8 & 800 \\
\hline 60 & 3 & 40 & 8 & 1,000 \\
\hline 60 & 3 & & & \\
\hline
\end{tabular}

Fuente: elaboración propia 
Como las encuestas fueron aplicadas en las zonas cercanas a los puentes peatonales de la ciudad, los juegos de elección fueron agrupados en tres bloques con el fin de presentar a cada individuo únicamente 4 situaciones de elección, evitando así la impaciencia y la fatiga de los individuos seleccionados, que sin duda, se hubiese presentado en caso de utilizar un número mayor de tratamientos, que implicaría necesariamente un mayor tiempo dedicado a responder la entrevista.

\subsection{Especificación del modelo}

El interés por incorporar variables que captan las percepciones, actitudes y otros componentes psicosociales en la modelación del comportamiento de los usuarios de sistemas de transporte ha llevado al empleo cada vez más generalizado de los modelos híbridos, siendo su principal desafío el de integrar dos enfoques de modelación en un mismo proceso.

El marco conductual para los modelos de elección con variables latentes [18-20] hace explícito el análisis de los factores psicológicos latentes con el propósito de obtener información acerca de la conducta individual que no puede deducirse de las preferencias reveladas. En este marco de análisis son identificados tres tipos de factores latentes: actitudes, percepciones y preferencias, que se pueden incluir en el modelo integrado como variables latentes psicométricas, de tal manera que no solo se mejora la comprensión del comportamiento del individuo, sino que mejora también el poder predictivo del modelo [8].

El modelo integrado que se utilizó para estudiar el proceso de elección del uso de puentes peatonales, presentado en este artículo, está compuesto por un modelo de elección discreta y un modelo de variables latentes, cuyo diagrama general es presentado en la figura 2. Todas las variables y coeficientes han sido presentados explícitamente en el diagrama general, así como los parámetros a estimar y los términos de error considerados en el modelo. Adicionalmente, se ha supuesto que la accidentalidad tiene un término de error que también debe ser estimado. 


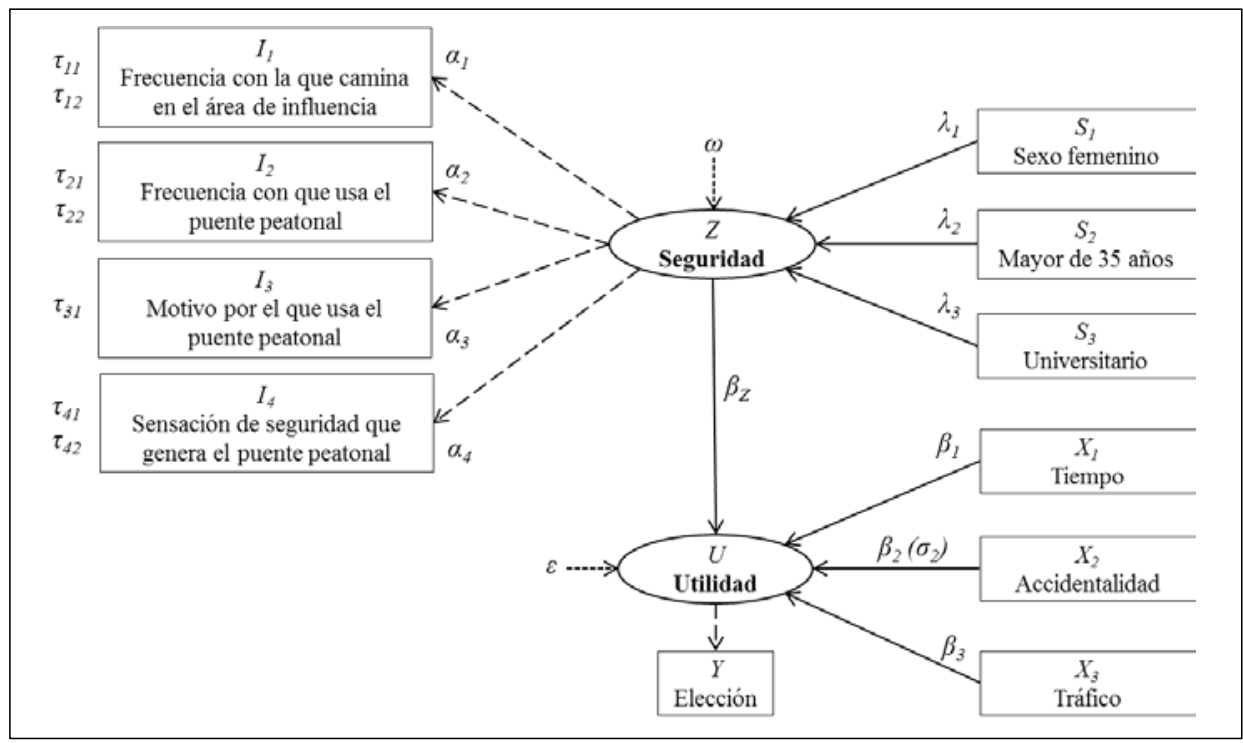

Figura 2. Modelo integrado de elección y variables latentes.

Fuente: elaboración propia

Ecuaciones estructurales

Para el modelo de variables latentes se condiciona la distribución de las variables latentes dadas las variables observadas. En este caso se especificó una función lineal para explicar la variable latente seguridad en función de tres atributos distintivos de los individuos: sexo, edad y nivel de estudios.

$$
Z=\lambda_{1} S_{1}+\lambda_{2} S_{2}+\lambda_{3} S_{3}+\omega, \omega \sim N\left(0, \sigma_{\omega}^{2}\right)
$$

Para el modelo de elección, se requiere la distribución de utilidades, dadas las variables observadas y la variable latente, en forma aditiva o en interacción. En el presente caso la variable latente seguridad se especificó en forma aditiva como se muestra.

$$
\mathrm{U}=A s c+{ }_{1}{ }_{1} \mathrm{X}_{1}+\left({ }^{2}{ }_{2}+\sigma_{2}\right) \mathrm{X}_{2}+{ }^{2}{ }_{3} \mathrm{X}_{3}+{ }^{2} \mathrm{Z} \mathrm{Z}+\mu, \mu \sim \operatorname{IIDGumbel}\left(0, \sigma_{\mu}^{2}\right)
$$

Como se ve en (2), la utilidad aleatoria ha sido descompuesta en una utilidad sistemática y en un término de error aleatorio, donde la utilidad sistemática es una función tanto de las variables observadas como de la variable latente. Igualmente, el coeficiente de $\mathrm{X}_{2}$, que en el modelo representa la diferencia en los indicadores de accidentalidad, ha sido especificado como un coeficiente aleatorio con media y desviación estándar $\sigma_{2}$. 


\section{Ecuaciones de medición}

Se utilizaron 4 ecuaciones de medición $(n=1,2,3,4)$, una por cada indicador en el modelo. Las ecuaciones de medición fueron especificadas con modelos de tipo Logit ordinal de 3 categorías $(k=1,2,3)$, a excepción del indicador 3 que solamente tiene dos categorías $(k=1,2)$. La notación general de estas ecuaciones para los indicadores que tienen tres categorías se presenta a continuación.

$$
\mathrm{P}\left(\mathrm{I}_{\mathrm{n}}=\mathrm{k}\right)=\frac{1}{1+\mathrm{e}^{-\left(\ddot{\mathrm{h}}_{\mathrm{k}}- \pm_{\mathrm{n}} \mathrm{Z}\right)}}-\frac{1}{1+\mathrm{e}^{-\left(\ddot{\mathrm{h}}_{\mathrm{k}-1}- \pm_{\mathrm{n}} \mathrm{Z}\right)}}
$$

Donde los $\tau_{n k}$ son parámetros a estimar, que representan el umbral de cada categoría $k$ e indicador $\mathrm{n}$ en el modelo ordinal, siendo $\tau_{n 0}=-\infty, \tau_{n 3}=+\infty$. Se puede ver fácilmente que la notación empleada sigue siendo válida para el indicador 3 que tiene dos categorías; solo basta hacer $\tau_{n 0}=-\infty, \tau_{n 2}=+\infty$.

Aquí sobresale la importancia de haber especificado los indicadores en forma creciente hacia la derecha, pues así resulta más natural la interpretación de los resultados asociados con la variable latente, a la vez que se puede verificar la correcta obtención de los umbrales para los indicadores que tienen tres categorías, caso en el cual se debe cumplir que $\tau_{n 2}>\tau_{n 1}$.

\subsection{Estimación}

La estimación del modelo se hizo con base en las diferencias de las variables de diseño, haciendo $X_{1}$ igual a las diferencias del tiempo y $X_{2}$ igual a las diferencias de accidentalidad. De esta manera fue posible fijar la función de utilidad de no usar el puente en 0 , especificando todas las variables en la función de utilidad de usar el puente, incluyendo la constante específica para esta alternativa.

El problema de estimación se resolvió mediante la técnica de máxima verosimilitud simulada, programando la función de verosimilitud conjunta (es decir, componente de elección y componente de variables latentes) en el paquete OxMetrics ${ }^{\mathrm{TM}}$ a partir de una adaptación del código escrito por [24]. El enfoque simultáneo utilizado supera los problemas de inconsistencia e ineficiencia que se pueden presentar cuando se emplea el enfoque secuencial [20], así como problemas asociados a valoraciones y pronósticos [25].

\section{RESULTADOS Y DISCUSIÓN}

El modelo híbrido de elección y variables latentes (ICLV) ha sido estimado con 19 parámetros: 4 parámetros $\beta$ y 1 parámetro $\sigma$ en el modelo de elección, 3 parámetros $\lambda$ 
en el modelo estructural, 4 parámetros $\alpha$ en los modelos de medición y 7 umbrales $\tau$ en los modelos ordinales que explican los indicadores por medio de la variable latente.

Los resultados son presentados en la tabla 4, donde se muestran los parámetros estimados para el modelo ICLV y para un modelo mixto (ML) de referencia. Los dos modelos se estimaron utilizando 1,000 extracciones para cada variable aleatoria y considerando el efecto de panel de las observaciones. La primera parte de la tabla presenta los parámetros estimados del modelo de elección; la segunda parte exhibe los parámetros del modelo estructural, y la última parte contiene los resultados del modelo de medición; al lado de cada coeficiente estimado se presenta el estadístico t-robusto. Aunque la tabla 4 no presenta los valores estimados para los umbrales, ya que a partir de ellos no se puede hacer ninguna interpretación práctica, se tuvo el cuidado de verificar su coherencia, comprobando para los indicadores de tres categorías que $\tau_{n 2}>\tau_{n 1}$.

Tabla 4. Modelos calibrados

\begin{tabular}{|c|c|c|c|c|c|}
\hline \multicolumn{2}{|r|}{ Modelo de elección } & \multicolumn{2}{|c|}{$I C V L$} & \multicolumn{2}{|c|}{$M L$} \\
\hline Coeficiente & Descripción & Est. & $\begin{array}{l}\text { Rob. } \\
\text { t-value }\end{array}$ & Est. & $\begin{array}{l}\text { Rob. } \\
\text { t-value }\end{array}$ \\
\hline Asc & Constante específica & $-0,832$ & $-2,01$ & -1.441 & -2.65 \\
\hline$\beta_{1}$ & Tiempo adicional & $-0,0354$ & $-3,41$ & -0.0442 & -4.75 \\
\hline$\beta_{2}$ & Media de la accidentalidad & 0,1928 & 1,97 & 0.203 & 1.92 \\
\hline$\sigma_{2}$ & Desviación de la accidentalidad & 0,000906 & 2,03 & 0.00302 & 4.46 \\
\hline$\beta_{3}$ & Tráfico & 0,000942 & 2,45 & 0.000856 & 2.82 \\
\hline$\beta_{z}$ & Variable latente seguridad & 0,975 & 2,43 & $\mathrm{n} / \mathrm{a}$ & $\mathrm{n} / \mathrm{a}$ \\
\hline$l(0)$ & Log-verosimilitud inicial & \multicolumn{4}{|c|}{-424.206} \\
\hline$l(\beta)$ & Log-verosimilitud en convergencia & \multicolumn{2}{|c|}{-388.317} & \multicolumn{2}{|c|}{-392.729} \\
\hline$L R$ & Razón de verosimilitud & \multicolumn{2}{|c|}{71.778} & \multicolumn{2}{|c|}{62.964} \\
\hline \multicolumn{6}{|c|}{ Modelo estructural - Variable latente seguridad } \\
\hline$\lambda_{1}$ & Sexo femenino & 0.546 & 2.20 & & \\
\hline$\lambda_{2}$ & Mayor de 35 años & 0.294 & 1.73 & & \\
\hline$\lambda_{3}$ & Universitario & 0.128 & 1.86 & & \\
\hline \multicolumn{6}{|c|}{ Modelo de medición } \\
\hline$\alpha_{1}$ & $\begin{array}{c}\text { Frecuencia con la que camina por } \\
\text { el área }\end{array}$ & 1.772 & 1.66 & & \\
\hline$\alpha_{2}$ & Frecuencia con la que usa el puente & 4.061 & 1.98 & & \\
\hline
\end{tabular}


Análisis de la percepción de seguridad en puentes peatonales: una aproximación mediante modelación híbrida 105

\begin{tabular}{|c|c|c|c|c|c|}
\hline \multicolumn{2}{|c|}{ Modelo de elección } & \multicolumn{2}{c|}{ ICVL } & \multicolumn{2}{c|}{ ML } \\
\hline Coeficiente & Descripción & Est. & $\begin{array}{c}\text { Rob. } \\
\text { t-value }\end{array}$ & Est. & $\begin{array}{c}\text { Rob. } \\
\text { t-value }\end{array}$ \\
\hline$\alpha_{3}$ & Motivo por el que usa el puente & 0.934 & 2.15 & & \\
\hline$\alpha_{4}$ & $\begin{array}{c}\text { Sensación de seguridad que genera } \\
\text { el puente }\end{array}$ & 9.211 & 1.63 & & \\
\hline \multicolumn{2}{|c|}{ Reporte general } & & \\
\hline $\mathrm{N}$ & Número de observaciones & \multicolumn{2}{|c|}{612} & & \\
\hline $\mathrm{R}$ & Número de realizaciones & \multicolumn{2}{c|}{1,000} & & \\
\hline
\end{tabular}

Fuente: elaboración propia

Lo primero que se evidencia es que el modelo híbrido tiene mayor calidad que el modelo mixto de referencia, ya que la inclusión de la variable latente mejora la log-verosimilitud en convergencia del modelo; también se mejora la significancia del coeficiente de la accidentalidad. La mejor calidad del modelo híbrido se puede verificar estadísticamente mediante la aplicación del test de razón de verosimilitud, que en este caso es $L R=8.824<\chi_{g . l=1 ; \alpha=0.05}^{2}$. Esto significa que el aumento de la log-verosimilitud el modelo híbrido es estadísticamente significativo, si se compara con el modelo de referencia, es decir, que en términos del mejor ajuste se justifica la inclusión de la variable latente.

El modelo de elección exhibe un comportamiento consistente, tanto para el modelo ICVL como para el modelo ML, ya que en ambos casos los signos de los coeficientes son razonables. Es correcto que el tiempo adicional, que resulta de la utilización del puente peatonal, tenga un efecto negativo en la función de utilidad, pues eso denota que, ceteris paribus, los individuos le conceden menos utilidad a la utilización del puente en la medida en que se incrementa la diferencia del tiempo con respecto al cruce de la calle.

Con respecto a la accidentalidad, también se considera correcto el signo obtenido. En este caso el signo positivo asociado a la diferencia de accidentalidad entre las dos opciones indica que las personas le asignan mayor utilidad al uso del puente peatonal cuando la diferencia del indicador de accidentalidad es mayor. Esto es razonable en la medida que los indicadores de accidentalidad asociados al uso del puente peatonal son menores a los que se mostraron en la encuesta de preferencias declaradas para la opción de no usar el puente. De todas formas, debido a la dificultad que posiblemente tuvieron los individuos a la hora de interpretar los valores de accidentalidad presentados en la encuesta, dicha variable se introdujo en el modelo suponiendo una variación aleatoria que, como se puede ver, resultó significativa. 
El coeficiente de la variable tráfico se obtuvo con signo positivo, lo que significa que en la medida en que el volumen de tráfico es mayor, manteniendo todo lo demás constante, los individuos le asignan mayor utilidad a la alternativa de usar el puente peatonal. Este comportamiento se considera coherente ya que a mayor tráfico será menos probable encontrar una brecha para cruzar la calle sin usar el puente peatonal. Ahora, con respecto a la variable latente, el hecho de haber obtenido un coeficiente positivo es consistente con la forma como fueron especificados los indicadores. Esto indica que los individuos que tienen una mayor percepción de seguridad le conceden mayor utilidad a la alternativa de usar el puente peatonal.

El modelo de medición muestra que la variable latente se manifiesta principalmente en el indicador que mide la sensación de seguridad que genera el puente, ya que su coeficiente, además de ser significativo al $10 \%$, tiene una magnitud que es 2.25 veces más grande que el coeficiente que le sigue en tamaño. Esto sugiere que la sensación de seguridad que genera el puente es el indicador más determinante desde la perspectiva de los usuarios. El modelo estructural muestra que la variable latente seguridad es mayor para las mujeres que para los hombres; asimismo, es mayor para las personas que tienen más de 35 años y es mayor también para los individuos que han alcanzado un nivel de estudios universitarios.

El modelo estructural deja ver entonces que el segmento de la población que le concede mayor valor a la seguridad y que por lo tanto tendría mayor disposición a usar los puentes peatonales está conformado por mujeres, mayores de 35 años que han alcanzado título universitario. En contraposición se tendría que las personas menos adeptas a usar los puentes peatonales serían los hombres menores de 35 años con nivel de estudios inferior al universitario. Aunque aparentemente podría existir una correlación entre la edad y el nivel de estudios, se hizo el cálculo del coeficiente de Pearson encontrando, para la muestra, un valor de 0.21 que no pone en riesgo el supuesto de independencia de estas variables.

\section{CONCLUSIONES}

Se estimó un modelo integrado de elección y variables latentes para estudiar la percepción de seguridad en el uso de puentes peatonales. Aunque en ciertos estudios de elección de transporte es común considerar atributos asociados al viaje de los individuos [26] en este caso se dio más importancia a los atributos del contexto en el que el individuo realiza su caminata, tales como los niveles de accidentalidad y los volúmenes vehiculares.

A pesar de que el modelo híbrido estimado se considera teórica y estadísticamente superior a un modelo mixto de referencia, su valor agregado en comparación con los 
modelos de elección convencionales es, por ahora, bastante limitado, especialmente en el análisis del efecto que políticas de intervención del sistema de transporte producen sobre la percepción de seguridad, puesto que la especificación de las ecuaciones estructurales consideró únicamente características socioeconómicas de los individuos, pero no factores objetivos.

Se logró evidenciar que la percepción de seguridad es determinante en la decisión de utilizar o no un puente peatonal cuando se cruza una vía. El modelo estructural permitió identificar que las mujeres mayores de 35 años con nivel de estudios universitarios conceden más importancia a la seguridad que otro tipo de individuos. Los resultados son contundentes en el sentido que las políticas para incrementar el uso de los puentes peatonales, con respecto al atributo seguridad, deben considerar principalmente un mejoramiento de las condiciones físicas de los puentes peatonales para incrementar la sensación de seguridad que genera el puente y consecuentemente aumentar su uso. Así mismo, las campañas educativas que se emprendan para motivar el uso de los puentes deberían hacer un mayor énfasis en las personas jóvenes, especialmente de sexo masculino que no han alcanzado el nivel de estudios universitario.

El modelo de elección determinó que el tráfico es una variable importante en la decisión y, aunque no se puede emplear como una variable de política sobre la cual se puedan tomar decisiones en este contexto, sí permite deducir que la probabilidad de que una persona decida usar un puente peatonal puede depender del período del día, ya que en las horas pico el flujo vehicular aumenta, haciendo más difícil cruzar la calle y por lo tanto, aumenta la probabilidad de usar dicho puente. Esta situación permite sugerir a las autoridades que ejecutan campañas de sensibilización, que es preferible hacer estas en los períodos valle, en lugar de tratar de hacerlas en los períodos de mayor afluencia vehicular y peatonal.

Del modelo de elección se concluye que el tiempo adicional requerido para utilizar el puente peatonal, en comparación con el tiempo de cruzar la calle a nivel, es un factor determinante de la decisión de usar el puente peatonal. Por tal razón, los puentes peatonales deben tener un acceso rápido y estar ubicados lo más cerca posible al lugar donde se congrega el mayor número de personas, logrando así reducir el tiempo de caminata y en consecuencia aumentando la probabilidad de uso del puente peatonal. Ahora bien, para aumentar el uso de puentes peatonales se podrían implementar barreras que hagan la caminata más larga; sin embargo, esto ocasiona una pérdida de utilidad que debería ser estudiada. 


\section{REFERENCIAS}

[1] R. Löhner, "On the modeling of pedestrian motion", Applied Mathematical Modelling 34 (2), pp. 366-382, 2010.

[2] C. Jakobsson, A. Gamble, T. Gärling, O. Hagman, M. Polk, D. Ettema, M. Friman, L. Olsson, "Subjective well-being related to satisfaction with daily travel", Transportation 38 (1), pp. $1-15,2011$.

[3] M. V. Johansson, T. Heldt, P. Johansson, "The effects of attitudes and personality traits on mode choice", Transportation Research Part A 40 (6), pp. 507-525, 2006.

[4] E. R. Dugundji, A. Páez, T. A. Arentze, J. L. Walker, “Transportation and social interactions”, Transportation Research Part A 45, pp. 239-247, 2011.

[5] A. Orro, "Modelos de elección discreta en transportes con coeficientes aleatorios", Tesis doctoral en Ingeniería Civil, Escuela Técnica Superior de Ingenieros de Caminos, Canales y Puertos, Universidad de A Coruña, A Coruña, abril de 2005.

[6] M. F. Yáñez, S. Raveau, J. D. Ortúzar "Inclusion of latent variables in Mixed Logit models: Modelling and forecasting”, Transportation Research Part A 44, pp. 744-753, 2010.

[7] A. Tsirimpa, A. Polydoropoulou, A. Constantinos, "Development of a Latent Variable Model to Capture the Impact of Risk Aversion on Travelers' Switching Behavior", Journal of Choice Modelling 3 (1), pp. 127-148, 2010.

[8] R. A. Daziano, "Taking account of the role of safety on vehicle choice using a new generation of discrete choice models", Safety Science, vol. 50, Issue 1, pp. 103-112, 2012.

[9] A. Daly, S. Hess, B. Patruni, D. Potoglou, C. Rohr, "Using ordered attitudinal indicators in a latent variable choice model: a study of the impact of security on rail travel behaviour", Transportation 39, pp. 267-297, 2012.

[10] C. Prato, S. Bekhor, C. Pronello, "Latent variables and route choice behaviour", Transportation, 39, pp. 299-319, 2012

[11] L. Márquez, V. Cantillo, J. Arellana, "How are comfort and safety perceived by inland waterway transport passengers?”, Transport Policy, vol. 36, pp. 46-52, 2014.

[12] C. R. Cherry, A. A. Adelakun, "Truck driver perceptions and preferences: Congestion and conflict, managed lanes, and tolls," Transport Policy 24 pp. 1-9, 2012.

[13] H. Wennberg, C. Hydén, A. Ståhl, "Barrier-free outdoor environments: Older peoples' perceptions before and after implementation of legislative directives", Transport Policy $17 \mathrm{pp}$. 464-474, 2010.

[14] A. Delbosc, G. Currie, "Modelling the causes and impacts of personal safety perceptions on public transport ridership", Transport Policy 24 pp. 302-309, 2012. 
[15] M. Riscanevo, E. Alonso, "Análisis experimental de los factores que determinan el uso de puentes peatonales en Tunja”, Trabajo fin de carrera, Ingeniería en Transporte y Vías, Universidad Pedagógica y Tecnológica de Colombia, Tunja, 2013.

[16] S. L. Moreno, “Muertes y lesiones por accidentes de transporte, Colombia, 2012”, Instituto nacional de medicina legal y ciencias forenses, Bogotá, 2013.

[17] Alcaldía Mayor de Tunja, "Caracterización de la movilidad", Convenio interadministrativo N. ${ }^{\circ} 010$ de 2012, Tunja, octubre 2012.

[18] M. Ben-Akiva, B. Boccara, "Integrated Framework for Travel Behavior Analysis”, presented at the International Association of Travel Behavior Research (IATBR) Conference, Aix-enProvence, France, 1987

[19] J. D. Ortúzar, L. G. Willumsen, Modelling transport, 4th edition, London, UK: Chichester: John Wiley \& Sons, 2011, 586 p.

[20] J. L. Walker, "Extended discrete choice models: Integrated framework, flexible error structures, and latent variables". Tesis doctoral, Massachusetts Institute of Technology, Cambridge, Massachusetts, 2001.

[21] K. Ashok, W. R. Dillon, S. Yuan, "Extending discrete choice models to incorporate attitudinal and other latent variables", Journal of Marketing Research 39 (1), 31-46, 2002.

[22] C. M. Rungie, L. V. Coote, J. J. Louviere, "Latent variables in discrete choice experiments", Journal of Choice Modelling 5 (3), pp. 145-156, 2012.

[23] J. Kim, S. Rasouli, H. Timmermans, "Hybrid Choice Models: Principles and Recent Progress Incorporating Social Influence and Nonlinear Utility Functions", Procedia Environmental Sciences 22, pp. 20-34, 2014.

[24] J. Arellana, Modelos de elección de hora de inicio de viajes, Tesis doctoral, Santiago, Chile: Pontificia Universidad de Chile, 2012.

[25] S. Raveau, R. Alvarez Daziano, M. F. Yáñez, D. Bolduc, J. D. Ortúzar, "Sequential and Simultaneous Estimation of Hybrid Discrete Choice Models: Some New Findings", Transportation Research Record 2156, pp. 131-139, 2010.

[26] J. J. Egaña, J. D. Ortúzar, L. Rizzi, “Inclusión de variables latentes en modelos de elección discreta para usuarios de transporte público interurbano", presentado en Cuarto Congreso Chileno de Ingeniería de Transporte, Concepción, octubre 2009. 